\title{
Neuro Endocrine Strategies during Adaptation to Stress
}

\author{
A. Bhimte ${ }^{1 *}$, N. Thakur ${ }^{1}$, V.P. Maurya ${ }^{2}$, B. Balamurugan ${ }^{3}$ and G. Singh ${ }^{2}$ \\ ${ }^{1}$ Animal Physiology Division, National Dairy Research Institute, \\ Karnal-132001, Haryana, India \\ ${ }^{2}$ Division of Physiology and Climatology, ${ }^{3}$ Division of Animal Reproduction, ICAR-Indian \\ Veterinary Research Institute, Izzatnagar- 243122, U.P., India \\ *Corresponding author
}

\section{Key words \\ Neuro endocrine strategies, Stress adaptation, Stress cascade}

\section{Article Info}

Accepted: 08 June 2018 Available Online: 10 July 2018

\section{A B S T R A C T}

The survival and well-being of all species requires appropriate physiological responses to environmental and homeostatic challenges. Disruptions in homeostasis (ie, stress) place demands on the body that are met by the activation of 2 systems, the hypothalamicpituitary-adrenal (HPA) axis and the sympathetic nervous system (SNS). Stressor-induced activation of the HPA axis and the SNS results in a series of neural and endocrine adaptations known as the "stress response" or "stress cascade." A challenge to homeostasis (a stressor) initiates the release of corticotropin-releasing hormone (CRH) from the hypothalamus, which in turn results in release of adrenocortiotropin hormone (ACTH) into general circulation. ACTH then acts on the adrenal cortex resulting in release of a species specific glucocorticoid into blood. Glucocorticoids act in a negative feedback fashion to terminate the release of $\mathrm{CRH}$. The body strives to maintain glucocorticoid levels within certain boundaries and interference at any level of the axis will influence the other components via feedback loops, so that the respective contribution of the neuroendocrine and autonomic systems is tuned in accordance with stressor modality or intensity. Limbic regions that are responsible for regulating stress responses intersect with circuits that are responsible for memory and reward, providing a means to tailor the stress response with respect to prior experience and anticipated outcomes.

\section{Introduction}

Stress is a state of threatened homeostasis caused by intrinsic or extrinsic adverse forces (stressors) and is counteracted by an intricate repertoire of physiologic and behavioral responses aiming to maintain/reestablish the optimal body equilibrium (eustasis) (Chrousos,2009). The adaptive stress response depends upon a highly interconnected neuroendocrine, cellular and molecular infrastructure, the stress system. Key components of the stress system are the hypothalamic-pituitary-adrenal (HPA) axis and the autonomic nervous system (ANS), which interact with other vital centers in the central nervous system (CNS) and tissues/organs in the periphery to mobilize a successful adaptive response against the imposed stressor(s) (Cota et al., 2007). 
Deregulation of the stress system (hyper- or hypo-activation) in association with potent and/or chronic stress can markedly disrupt the body homeostasis leading to a state of cacostasis or allostasis, with a spectrum of clinical manifestations.

There are many steps involved in the initial perception of a stress and the stimulation of an adequate response that will bring about the regulation of homeostasis (Chen etal. 2015). Moberg and Mench (2000) outlined the biological model known as the general adaptation syndrome that animals have developed in order to cope with a perceived stress.

The perception of stress requires a change in the biological function of the animal in order to cope with and reduce the negative effects associated with a stress response (Figure 1b). The achievement of regaining homeostasis results in the normal biological function of the animal being restored (Figure 1a) (Lynch 2010).

Due to the fact that the SAM(sympathetic adrenal medullary system) is activated in response to a short-term or acute stress, its inability to rectify a stressful event results in the activation of the HPA axis (Lynch 2010), which, as mentioned earlier, is involved in resolving long-term, chronic stress (Kumar et al., 2012). Figure 2 illustrates the HPA axis pathway, associated hormones and target systems.

\section{Stress system - physiology and interactions}

Neuroendocrine effectors of the stress response: "The Stress System"

Although the entire CNS is directly or indirectly involved in preserving the overall body homeostasis, specific areas of the brain have critical, distinct roles in orchestrating the stress response (Aguilera et al., 2008). As such, the central components of the stress system are located in the hypothalamus and the brainstem and include the parvocellular corticotropin-releasing hormone (CRH) and arginine vasopressin (AVP) neurons of the paraventricular nuclei (PVN) of the hypothalamus, and the $\mathrm{CRH}$ neurons of the paragigantocellular and parabranchial nuclei of the medulla, as well as the locus coeruleus (LC) and other catecholaminergic, norepinephrine (NE)-synthesizing cell groups of the medulla and pons (central sympathetic nervous system). The peripheral limbs of the hypothalamic-pituitary-adrenal (HPA) axis, together with the efferent sympathetic/adrenomedullary system, constitute the peripheral components of this interconnected system (Hirsch et al., 2012).

\section{Central stations of the stress system - CRH, AVP and catecholaminergic neurons}

The central neurochemical circuitry responsible for the stress system activation forms a physiological system within the CNS, consisting of both stimulatory and inhibitory networks with multiple sites of interaction which modulate and fine-tune the adaptive stress response (Rotzinger et al., 2010). The key components of these networks are the hypothalamic CRH and AVP neurons in combination with the central catecholaminergic (LC/NE) neurons (Figure 2).

The central stress system activation is based on reciprocal reverberatory neural connections between the PVN CRH and the catecholaminergic LC/NE neurons, with $\mathrm{CRH}$ and NE stimulating the secretion of each other through CRH receptor-1 (CRH-R1) and $\alpha 1$ noradrenergic receptors, respectively(Young et al., 2009). Autoregulatory ultrashort negative feedback loops exist in both the PVN $\mathrm{CRH}$ and the brainstem catecholaminergic 
neurons, with collateral fibers inhibiting $\mathrm{CRH}$ and catecholamine secretion respectively, via inhibition of the corresponding presynaptic $\mathrm{CRH}-$ and $\alpha 2$-noradrenergic receptors (Veldhuis et al., 2011). In addition, multiple other regulatory central pathways exist, since both $\mathrm{CRH}$ and catecholaminergic neurons receive stimulatory innervation from the serotoninergic and cholinergic systems, and inhibitory input from the gamma-aminobutyric acid (GABA)/benzodiazepine (BZD) and the opioid neuronal systems of the brain, as well as by glucocorticoids (the end-product of the HPA axis) (Lutfy et al., 2011).

Stressful stimuli may reach the central nervous system through somato- or viscerosensory pathways through spinal or brainstem sensory neurons (Fig. 3). Somatosensory signals are detected by noxious, mechanical, thermosensitive, etc., or specific (photic, acoustic, taste, equilibral) receptors and carried by spinal and cranial sensory nerves (Chen et al., 2004). Viscerosensory signals arise from the body and may reach spinal and supraspinal receptors by neural (from interoceptors) or humoral pathways. (Accordingly, stressful stimuli have been previously classified as neurogenic and systemic stressors).

Stress responses in general can be divided into short and long circuit categories.

Short circuit mechanisms are also called spinal stress responses based on spinal reflexes, while the "long circuits" are also called supraspinal stress responses. The maintenance of homeostasis requires precise coordination of autonomic, neuroendocrine, and behavioral responses to contend with constant perturbations of the internal and external environments (Du et al., 2009). Therefore, the long circuits include higher centers such as the neuroendocrine hypothalamus, the limbic system, and the cerebral cortex. Each of them is neuronally connected with brainstem and spinal somato- and viscerosensory centers, and they are also interconnected with each other (Fig. 4). The output system (i.e., the realization of the stress response) involves two major routes: neuronal and neuroendocrine (McMaster et al., 2011). The neuronal responses are carried by either somatomotor or visceromotor (autonomic) fibers in cranial or peripheral nerves. Thus, both motor and autonomic stress responses finally arise from brainstem or spinal neurons.

\section{Hypothalamic-Pituitary-Adrenal axis}

(HPA)

The HPA axis is a vital component of both the central and the peripheral limb of the stress system. The HPA axis can be regarded as a crucial neuroendocrine system that is involved in the control of diverse physiological processes and adaptations to stress (Mormède et al., 2007). When the environmental pressure of a perceived stress exceeds that to which an animal's adaptive mechanisms can accomodate (Kumar et al., 2012), this system produces energetic metabolites that arise either from energy storage tissues or from the transformation of proteins into energetic metabolites (Mormède et al., 2011). As such, HPA axis integrity and precise regulation of its function are essential characteristics of the successful adaptive response to any stressor. At the level of the hypothalamic-pituitary unit, CRH is released into the hypophyseal portal system and acts as the principal regulator of the anterior pituitary ACTH secretion.

\section{The stress response}

Following disruption of homeostasis, the HPA axis and the SNS are activated. These constitute the essential elements mediating the stress response, all of which are directed towards homeostatic preservation. The stress responses related to the SNS are covered in a 
separate report in this supplement (McEwen et al., 2007). The HPA axis meets the demands of stress primarily through the synthesis and/or just release of 3 key hormones, corticotropin-releasing hormone (CRH), adrenocorticotropin hormone $(\mathrm{ACTH})$, and a species-specific glucocorticoid, either cortisol (COR) (human, nonhuman primate, swine, and dog) or corticosterone (CORT) (rodents). Other key elements in the stress response are the organs (Fig. 5) that release and are acted upon by these hormones. These include the hypothalamic and hippocampal areas of the brain, the anterior pituitary, and the adrenal gland.

This sensitization of the HPA axis could actually be considered advantageous for survival because more efficient responding occurs. Continued excretion at elevated levels, however, is likely to be detrimental because glucocorticoid excess would be the ultimate outcome (Refojo et al., 2011). Understandings of the cellular and molecular changes that control sensitization of the HPA axis are important because pathological sensitization can play a major role in both depression and post-traumatic stress disorders. Further, there is evidence that even a single highly traumatic event may produce protracted neuroendocrine sensitization to stress, relative roles of $\mathrm{CRH}$ and AVP in the stress response (Lkhagvasuren et al., 2011). CRH plays a pivotal integrative role in the response to stress that encompasses initiation, modulation, and inhibition of the stress response. In fact, giving $\mathrm{CRH}$ to rats will produce most of the signs associated with exposure to a stressor. CRH may serve as a gatekeeper of the stress response as it is subject to negative feedback on several fronts. Cortisol as well as norepinephrine and gamma-aminobutyric acid shut off the production of CRH (Nakamura et al., 2011). Activation of the $\mathrm{CRH}$ receptor leads to the release of ACTH from the pituitary into the general circulation and the ultimate release of glucocorticoids from the adrenal cortex. However, the presence of this neuropeptide and its receptors in the neurons of other brain areas and in organs as diverse as skin, heart, and gastrointestinal tract suggest a multiplicity of functions for $\mathrm{CRH}$.

Glucocorticoids play a crucial role in the regulation of the basal HPA axis activity and in the termination of the stress response by acting at multiple levels, including extrahypothalamic regulatory centers, the hypothalamus and the pituitary (Schulz et al., 2012). As such, the inhibitory glucocorticoid feedback on the ACTH secretory response limits the duration of the total tissue exposure to glucocorticoids, thus minimizing the catabolic, anti-reproductive and immunosuppressive effects of these hormones.

\section{Sympathetic/adrenomedullary and parasympathetic systems}

The autonomic nervous system (ANS) provides a rapidly responsive mechanism to control a wide range of physiologic functions. As such, the cardiovascular, respiratory, gastrointestinal, renal, endocrine and other vital systems are tightly regulated by either the SNS or the parasympathetic system or the combined activity of both (Gilbey et al., 1993). Indeed, the ANS activity is typically regulated through a dual reaction mechanism, since the parasympathetic system can equally assist or antagonize most of the SNS functions by withdrawing or increasing its activity, respectively.

Sympathetic innervation of peripheral organs is derived from the efferent preganglionic fibers whose cell bodies lie in the intermediolateral column of the spinal cord. These nerves synapse in the bilateral chain of sympathetic ganglia with postganglionic sympathetic neurons, which innervate the smooth muscle cells of the vasculature, 
skeletal muscles, heart, kidneys, gut, adipose tissue and many other organs (Burnstock et al., 1989). The preganglionic neurons are primarily cholinergic, whereas the postganglionic neurons release mostly noradrenaline. The SNS activity has an additional humoral contribution consisting of circulating epinephrine and, to a lesser extent, norepinephrine released by the adrenal medulla which can be considered as a modified sympathetic ganglion.

\section{Stress system - Interactions with other CNS components}

The stress system not only sets the arousal level and regulates vital signs, but further interacts with other crucial CNS components, including the mesocorticolimbic dopaminergic system ("reward" system), the amygdala/hippocampus complex and the arcuate nucleus POMC neuronal system (Gray et al., 1989). In turn, following activation by stress, these CNS systems act via specific neuronal pathways to modify the stress system activity, hence forming a complex reciprocal mechanism which fine-tunes the adaptive response. Of note, well-established interactions exist between the stress system and distinct CNS centers which are essential for survival, such as the thermoregulatory and appetite-satiety centers.

\section{Mesocorticolimbic dopaminergic system}

The mesocortical and mesolimbic components of the dopaminergic system are highly innervated by PNV CRH neurons and the LC/NE-sympathetic noradrenergic system and, thus, are activated by $\mathrm{CRH}$, catecholamines and glucocorticoids during stress. The mesocortical system contains dopaminergic neurons of the ventral tegmentum which send projections to the prefrontal cortex. Activation of these neurons appears to centrally suppress the stress system response and is implicated in anticipatory phenomena and cognitive functions.

\section{Amygdala/Hippocampus}

$\mathrm{CRH}$ fibers also interconnect the amygdala with the bed nucleus of the stria terminalis and the hypothalamus. Conversely to the stimulatory $\mathrm{CRH}$ and norepinephrine effect, the hippocampus exerts a tonic and stimulated inhibitory effect on the amygdala activity and the PVN $\mathrm{CRH}$ and LC/NE-sympathetic systems. Indeed, the hippocampus plays an important role in shutting off the HPA stress response. Hippocampal atrophy or damage impairs this shut off function and can lead to prolonged HPA responses to psychological stressors (McEwen et al., 2007). These findings led to the "glucocorticoid cascade hypothesis" of stress and aging.

\section{Arcuate Nucleus Proopiomelanocortin (POMC) Neuronal System}

Stress system activation stimulates hypothalamic release of POMC-derived peptides, including $\alpha$-melanocyte-stimulating hormone $(\alpha-\mathrm{MSH})$ and $\beta$-endorphin, which reciprocally inhibit the activity of both the central stress system components (Refojo et al., 2011). Moreover, through projections of these neurons to the hind brain and the spinal cord, "stress- induced analgesia" is achieved by inhibition of the ascending pain pathways.

\section{CLOCK system}

More recently, it became evident that the stress system is interconnected and communicates at multiple levels with an additional vital system, defined as the CLOCK system, which generates the body circadian rhythms and regulates a wide range of physiologic functions (Nader et al., 2010). This system is comprised by a main central hypothalamic component and numerous 
associated extra-hypothalamic, peripheral components. The central CLOCK system component is located in the suprachiasmatic nuclei (SCN) of the hypothalamus and acts as a "master" CLOCK under the influence of light/dark input through the eyes. Light/dark information can travel through the retinohypothalamic tract (RHT; a photic neural input pathway implicated in the regulation of circadian rhythms in mammals) (Fig. 6-8).

\section{Stress system - Endocrine axes interactions}

The stress system is tightly interconnected with all the major endocrine axes, including the reproductive, growth and thyroid axis. This ensures that the activity of the endocrine system is rapidly regulated in a coordinated and precise way in order to serve the adaptive stress response and maximize the chances of survival against the imposed stressor(s).

\section{Stress system - Reproductive axis}

Although the observation that stress can impact negatively on the reproductive function traces back to antiquity, the exact pathophysiologic and molecular mechanisms which mediate this effect still pose a research challenge (Chand et al.,2011).

The reproductive system, both in females and males, is inhibited at all levels by various components of the HPA axis (Figure 9). As such, CRH suppresses the gonadotropinreleasing hormone $(\mathrm{GnRH})$ neurons directly and indirectly via enhancing $\beta$-endorphin secretion by the arcuate POMC neurons.

\section{Stress system - Growth axis}

The growth axis is also inhibited at various levels during stress (Figure 9). Prolonged activation of the HPA axis leads to suppression of growth hormone $(\mathrm{GH})$ secretion and inhibition of somatomedin $\mathrm{C}$ $(\mathrm{SmC})$ and other growth factor effects on their target tissues by glucocorticoids (Vamvakopoulos et al., 1998), presumably via inhibition of the c-jun/c-fos heterodimer. However, acute transient elevations of $\mathrm{GH}$ concentrations in plasma may occur at the onset of the stress response, as well as after acute administration of glucocorticoids, potentially mediated through GRE-stimulated GH expression (Casanueva et al., 1990).

In addition to the direct effects of glucocorticoids which play a key role in the suppression of growth observed under prolonged stress, increased somatostatin (STS) secretion caused by CRH which results in inhibition of $\mathrm{GH}$ secretion appears to also contribute to the stress-related suppression of the growth axis (Figure 9). Redirection of oxygen, nutrients and vital substrates to the brain and other stressed organ/tissues where they are needed most in the context of the adaptive stress response is the apparent teleology for the suppressive effects of stress on growth

\section{Stress system - Thyroid axis}

Stress-related inhibition of thyroid axis activity has also been documented (Figure 10). Chronic HPA axis activation is associated with decreased production of thyroid stimulating hormone (TSH) and inhibited conversion of the relatively inactive thyroxine (T4) to the more biologically active triiodothyronine (T3) in peripheral tissues (a condition described as the "euthyroid sick" syndrome) (Roelfsema et al., 2009). Although the exact mechanism(s) underlying these effects have not been fully clarified, increased circulating glucocorticoid levels are considered to mediate the stress-induced suppression of the thyroid axis which serves desired energy conservation during the adaptive stress response. 
Figure.1 The stages involved in the biological general adaptation syndrome in response to stress in animals

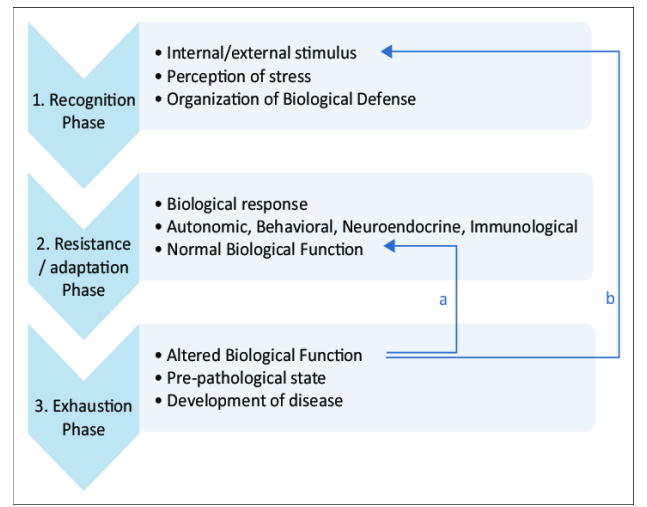

\section{Stress system - physiology and interactions}

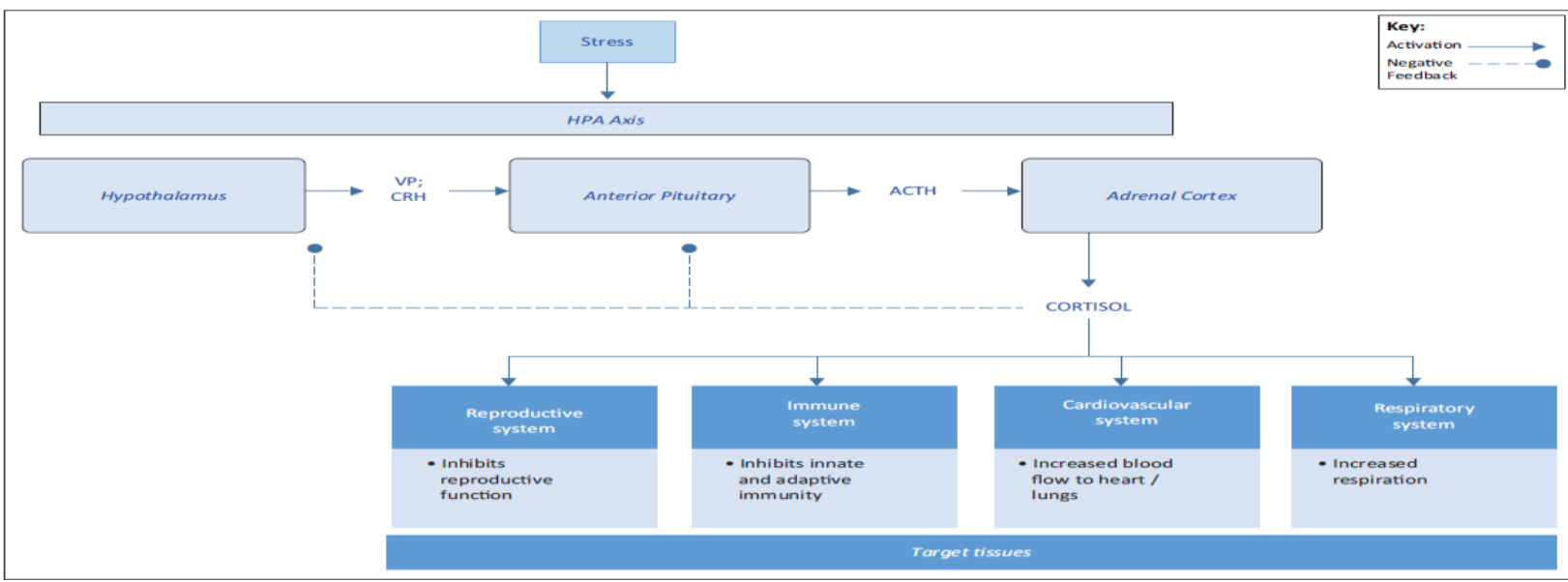

Figure.2 A simplified representation of the central and peripheral components of the stress system

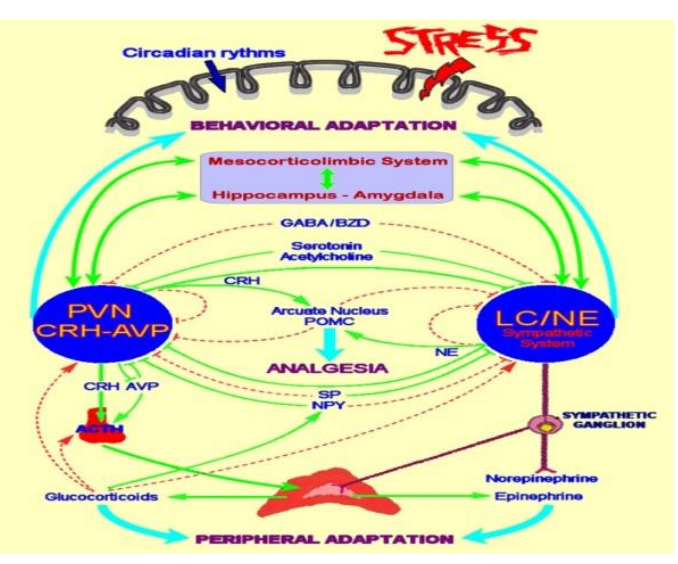


Figure.3 Spinal or brainstem sensory neurons

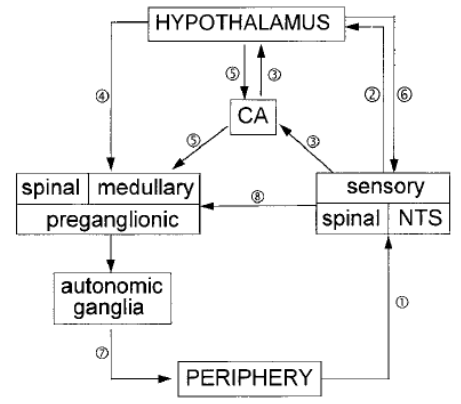

Figure.4 Stress responses short and long circuit categories

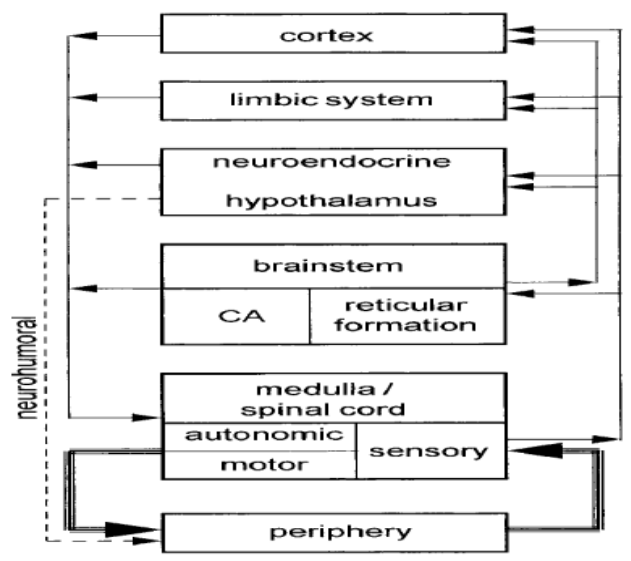

Figure.5 Hypothalamic-Pituitary-Adrenal (HPA) axis

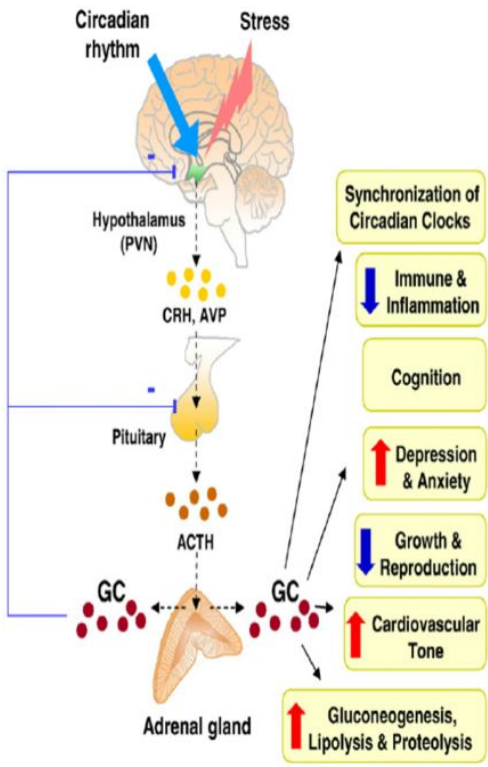


Figure.6 Projections from the central nucleus of the amygdala to the hypothalamus and to the central autonomic system

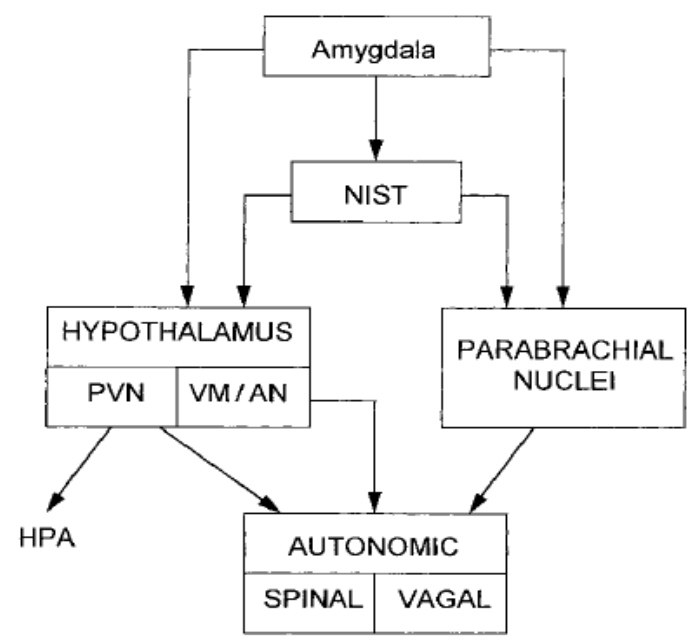

Figure.7 Central CLOCK synchronizes the peripheral CLOCKs and regulates located peripheral organ activities via neural and humoral interactions

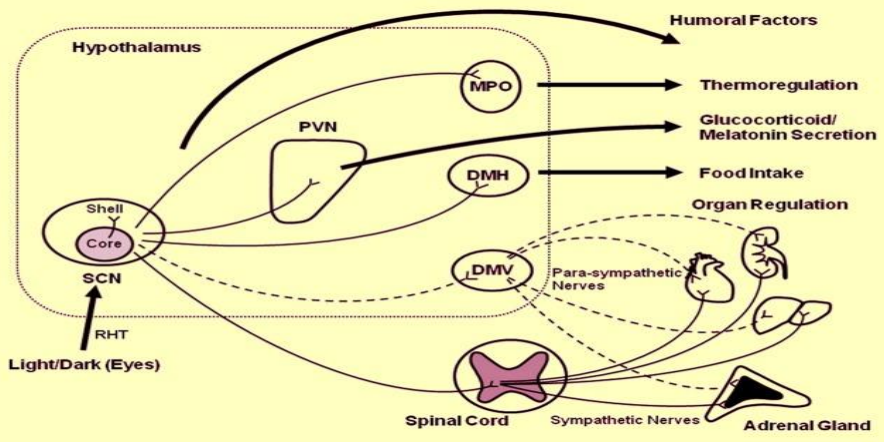

Figure.8 The light-activated central CLOCK in the suprachiasmatic nucleus $(\mathrm{SCN})$

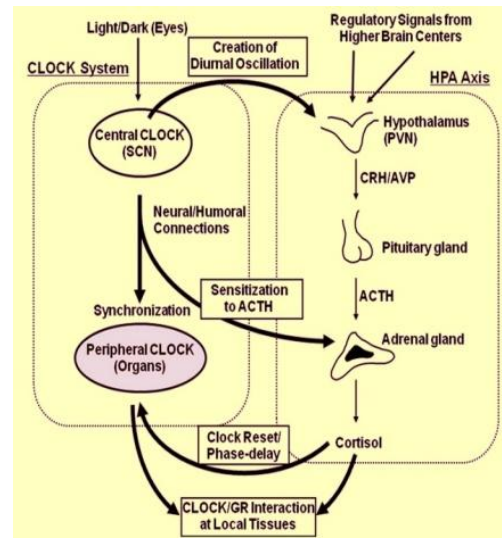

Figure.9 Schematic representation of the interactions between the hypothalamic-pituitaryadrenal (HPA) axis and the reproductive and growth axes

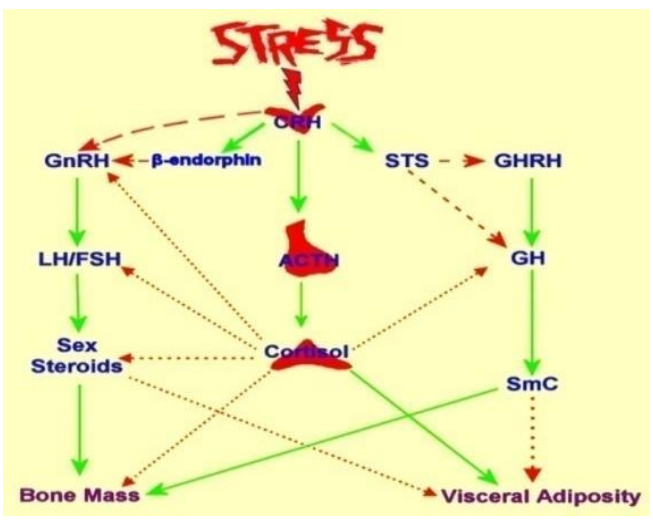


Figure.10 Schematic representation of the interactions between the hypothalamic-pituitaryadrenal (HPA) axis and the thyroid and immune function

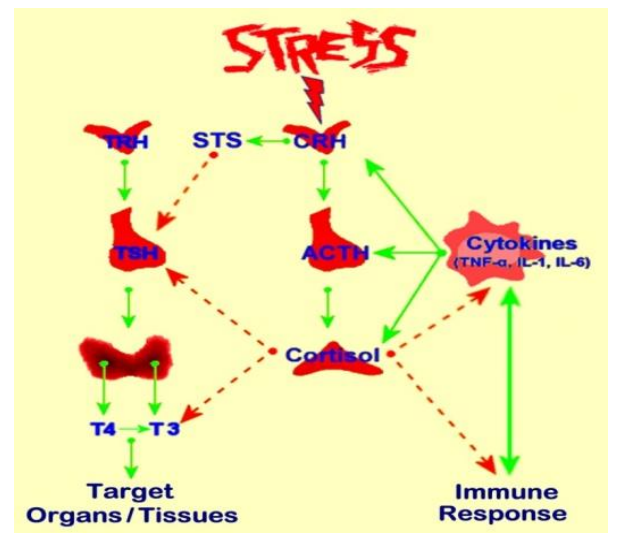

\section{Effects of thermal environment on appetite}

It makes sense that an individual adapts to changes in thermal environment by adjusting energy expenditure to maintain normal body temperature (see section on thyrotrophic axis above). At the molecular level, however, we are far from understanding the complex neuroendocrine mechanisms that occur during episodes of climatic change (Chrousos et al., 2000). The suppressive effect of heat stress on appetite is well recognized, but virtually nothing is known about the underlying endocrine mechanisms. The limited information that exists about neuroendocrineappetite interactions during thermal stress relates to cold exposure. Since the appetite regulators leptin and NPY also have metabolic effects, responses of the leptinNPY axis to ambient temperature are of particular interest. Recent work suggests that a response in NPY expression to hypothermia depends on the duration of exposure, but hypothermia consistently decreases leptin expression.

\section{Effects of generalized stress (induction of HPA axis) on appetite}

$\mathrm{CRH}$, produced in the hypothalamus, has a direct effect of decreasing feed intake in both normal and NPY-deficient mice (NPY -/- knockout), suggesting that this effect is not mediated through NPY (Kyrou et al.,2007). Leptin treatment increases hypothalamic CRH content and decreases appetite in rats. Glucocorticoids increase plasma leptin levels in rats. It is postulated that the increased leptin secretion does not decrease feed intake exclusively through decreasing NPY levels, but by increasing sensitivity to $\mathrm{CRH}$. There are receptors for $\mathrm{CRH}$ within the ventromedial hypothalamus $(\mathrm{VMH})$, an area which is rich in leptin receptors.

In conclusion it is apparent that numerous stressors arising from both endogenous and exogenous aspects of the animal production cycle have potentially inhibiting effects on overall productivity and well-being. The HPA axis functions as a coping mechanism to stress and re-adjusts an animal's homeostatic state by increasing secretion of stress-related hormones and bringing about a behavioural, autonomic, endocrine or immune response (Kumar et al., 2012) to enable the animal to cope with the perceived stress.

\section{References}

Aguilera, G., Subburaju, S., Young, S. and Chen, J.2008. The parvocellular vasopressinergic system and responsiveness of the hypothalamic 
Pituitary adrenal axis during chronic stress. Prog Brain Res. 170: 29-39.

Burnstock, G. 1989. Eur. Heart J. 10:15-21

Chrousos, G.P.2000. The role of stress and the hypothalamic-pituitary-adrenal axis in the pathogenesis of the metabolic syndrome: neuro-endocrine and target tissue-related causes. Int. J. Obes. Relat. Metab. Disord. 2 Suppl 2:S50-S55.

Casanueva, F.F., Burguera, B., Muruais, C. and Dieguez, C.1990. Acute administration of corticoids: a new and peculiar stimulus of growth hormone secretion in man. J Clin Endocrinol Metab. 70:234-237.

Chand, D. and Lovejoy, D.A. 2011. Stress and reproduction: controversies and challenges. Gen Comp Endocrinol. 171:253-257.

Chen, J.L., Villa, K.L., Cha, J.W., So, P.T.C., Kubota, Y. and Nedivi, E. 2012. Clustered dynamics of inhibitory synapses and dendritic spines in the adult neocortex. Neuron.74: 361-373.

Chrousos, G.P. 2009. Stress and disorders of the stress system. Nat Rev Endocrinol. 5(7):374-81.

Cota, D, Steiner, M.A., Marsicano, G., Cervino, C., Herman, J.P. and Grubler,Y. 2007. Requirement of cannabinoid receptor type 1 for the basal modulation of hypothalamicpituitary-adrenal axis function. Endocrinology.148:1574-1581.

Chen, W., Dang, T., Blind, R.D., Wang, Z., Cavasotto, C.N., Hittelman, A.B., Rogatsky, I., Logan, S.K. and Garabedian, 2008. Glucocorticoid receptor phosphorylation differentially affects target gene expression. Mol Endocrinol. 22:1754-1766.

Du, J., Wang, Y., Hunter, R., Wei, Y., Blumenthal, R. and Falke,C. 2009. Dynamic regulation of mitochondrial function by glucocorticoids. Proc Natl Acad Sci U S A 2009; 106:3543-3548.

Gilbey, M.P.1997. Fundamental aspects of the control of sympathetic preganglionic neuronal discharge. In: Jordan D, editor. Central Nervous Control of Autonomic Function. The Netherlands: Harwood Academic Publishers; pp. 1-28.

Gray, T.S., Carney, M.E. and Magnuson, D.J.1989. Direct projections from the central amygdaloid nucleus to the hypothalamic paraventricular nucleus: possible role in stress-induced adrenocorticotropin release. Neuroendocrinology. 50:433-446.

Hirsch, D. and Zukowska, Z. 2012. NPY and Stress 30 Years Later: The Peripheral View. Cell Mol Neurobiol.

Kumar, B., Manuja, A. and Aich, P. 2012. 'Stress and its impact on farm animals', Frontiers in Bioscience (Elite Ed.). $\quad 1$ 1: 1759-1767. https://doi.org/10.2741/e496

Lkhagvasuren, B., Nakamura, Y., Oka, T., Sudo, N. and Nakamura, K. 2011. Social defeat stress induces hyperthermia through activation of thermoregulatory sympathetic premotor neurons in the medullary raphe region. Eur J Neurosci. 34:14421452.

Kyrou, I. and Tsigos, C. 2007. Stress mechanisms and metabolic complications. Horm Metab Res. 39(6):430-8.

Lutfy, K., Aimiuwu, O., Mangubat, M., Shin, C.S., Nerio, N., Gomez, R., Liu, Y. and Friedman, T.C. 2011. Nicotine stimulates secretion of corticosterone via both $\mathrm{CRH}$ and AVP receptors. J Neurochem.

Lynch, E.M. 2010.Characterization of physiological and immune-related biomarkers of weaning stress in beef cattle, Doctoral dissertation, 
Department of Biology and National Institute for Cellular Biotechnology, National University of Ireland Maynooth.

McEwen, B.S. 2007. Physiology and neurobiology of stress and adaptation: central role of the brain. Physiol Rev. 87:873-904.

McMaster, A., Jangani, M., Sommer, P., Han, N., Brass, A. and Beesley, S. 2011. Ultradian cortisol pulsatility encodes a distinct, biologically important signal. PLoS One. 6:e15766.

Mormède, P., Andanson, S., Aupérin, B., Beerda, B., Guémené, D. and Malmkvist, J. 2007. 'Exploration of the hypothalamic - Pituitary - Adrenal function as a tool to evaluate animal welfare', Physiology \& Behavior. 92:317-339.

Moberg, G. and Mench, A. 2000. The biology of animal stress: Basic principles and implications for animal welfare, CABI, Wallingford, United Kingdom.

Mormede, P., Foury, A., Barat, P., Corcuff, J.B., Terenina, E. and Marissal-Arvy, N. 2011. 'Molecular genetics of hypothalamic - Pituitary - Adrenal axis activity and function', Annals of the New York Academy of Sciences. 1220: 127-136

Nader, N., Chrousos, G.P. and Kino T. 2010. Interactions of the circadian CLOCK system and the HPA axis. Trends Endocrinol Metab. 21:277-286.

Nakamura, K. 2011. Central circuitries for body temperature regulation and fever. Am J Physiol Regul Integr Comp
Physiol. 301:R1207-R1228

Refojo, D., Schweizer, M., Kuehne, C., Ehrenberg, S., Thoeringer, C. and Vogl, A.M. 2011.Glutamatergic and dopaminergic neurons mediate anxiogenic and anxiolytic effects of CRHR1. Science. 333:1903-1907.

Rotzinger, S., Lovejoy, D.A. and Tan, L.A. 2010. Behavioral effects of neuropeptides in rodent models of depression and anxiety. Peptides. 31: 736-756

Schulz, S. and Laessle, R.G. 2012. Stressinduced laboratory eating behavior in obese women with binge eating disorder. Appetite. 58:457-461.

Yang, S., Liu, A., Weidenhammer, A., Cooksey, R.C., McClain, D., Kim, M.K., Aguilera, G., Abel, E.D. and Chung, J.H. 2009. The role of mPer2 clock gene in glucocorticoid and feeding rhythms. Endocrinology. 150:2153-2160.

Vamvakopoulos, N.C. and Chrousos, G.P. 1994. Hormonal regulation of human corticotropin-releasing hormone gene expression: implications for the stress response and immune/inflammatory reaction. Endocr Rev. 15:409-420.

Veldhuis, J.D., Iranmanesh, A., Roelfsema, F., Aoun, P., Takahashi, P. and Miles, J.M. 2011. Tripartite control of dynamic ACTH-cortisol dose responsiveness by age, body mass index, and gender in 111 healthy adults. J Clin Endocrinol Metab. 96:2874-2881.

\section{How to cite this article:}

Bhimte, A., N. Thakur, V.P. Maurya, B. Balamurugan and Singh, G. 2018. Neuro Endocrine Strategies During Adaptation to Stress. Int.J.Curr.Microbiol.App.Sci. 7(07): 1132-1143. doi: https://doi.org/10.20546/ijcmas.2018.707.137 